\title{
AN OUTPUT CONTROLLABILITY PROBLEM FOR SEMILINEAR DISTRIBUTED HYPERBOLIC SYSTEMS
}

\author{
E. ZERRIK, R. LARHRISSI AND H. BOURRAY \\ MACS Group - AFACS UFR \\ Moulay Ismail University, Faculty of Sciences \\ Meknes, Morocco \\ e-mail: $\{$ zerrik3, hbourrayh@yahoo.fr\}
}

\begin{abstract}
The paper aims at extending the notion of regional controllability developed for linear systems to the semilinear hyperbolic case. We begin with an asymptotically linear system and the approach is based on an extension of the Hilbert uniqueness method and Schauder's fixed point theorem. The analytical case is then tackled using generalized inverse techniques and converted to a fixed point problem leading to an algorithm which is successfully implemented numerically and illustrated with examples.
\end{abstract}

Keywords: distributed parameter systems, semilinear hyperbolic systems, constrained controllability, fixed point.

\section{Introduction}

Distributed parameter systems are dynamical systems whose states depend not only on time but also on spatial coordinates. They are frequently encountered in practical engineering problems. Examples of a thermal nature are furnaces for heating metal slabs or heat exchangers, examples of a mechanical nature are large flexible aircrafts and robot arms, examples of an electrical nature are energy transmission lines.

Appropriate mathematical modeling of distributed parameter systems yields most often nonlinear partial differential equations. Clearly, such models involve using very sophisticated mathematical methods, but this enables us to describe the process more accurately and to implement more effective control strategies. Early lumping, which means the approximation of a nonlinear systems by linear ordinary differential equations of possibly high order with excessive simplifications, may completely mask the distributed nature of the system and therefore it is not always satisfactory.

For the last decade, semilinear distributed parameter systems have occupied an important place in control and systems theories. The study of this kind of systems has become fairly common, and is now an established area of research with an extensive and long list of publications and conference communications. For an extensive list of publications, see, e.g., (Klamka, 1991; Klamka, 2001;
Klamka, 2002) and the references therein, where sufficient conditions for constrained exact controllability in a prescribed time interval for semilinear dynamical systems in which the nonlinear term is continuously Frechet differentiable are formulated and proved assuming that the controls take values in a convex and closed cone with vertex at zero. The method used covers a wide class of semilinear abstract dynamical systems and is specially useful for semilinear ones with delays. For a controllability problem when one is faced with the question of steering a system from an initial state to a prescribed one, it is important to take into account the effects of nonlinearity. For example, Fabre et al. (1995) prove approximate controllability in $L^{p}(\Omega)$ for $1 \leq p<+\infty$ by means of a control which can be internal or on the boundary and when the nonlinearity is globally Lipschitz. That is achieved for some $\sigma>0$ and $\beta>0$ such that

$$
\|\mathcal{N}(s)\| \leq \sigma|s|+\beta .
$$

Moreover, in the case of the interior control, they prove approximate controllability in $\mathcal{C}_{0}(\Omega)$. The technique applied combines a variational approach to the controllability problem for a linear equation and fixed point methods.

Null-controllability was also proved in (FernándezCara, 1997) for semilinear distributed parabolic systems when the nonlinear term $\mathcal{N}(s)$ grows slower than $s \log |s|$ 
as $|s| \rightarrow 0$, or

$$
|| \mathcal{N}(s)|| \leq \varepsilon|s| \log |s| \text { for large }|s|,
$$

where $\varepsilon$ depends on the system domain, the final time and the geometric support of the control. Fixed point theorems and Gronwall's inequality remain important tools used by mathematicians to solve the various questions, particularly the controllability problem for semilinear systems, see (Zuazua et al, 1990; Kassara et al, 1983) and the references therein. Such a notion has been treated for situations where the subregion $\omega$ of interest is the interior to $\Omega$. These questions are particularly natural in a setting of the wave equation where a finite propagation speed excludes exact controllability of such a system.

The notion of regional controllability for distributed linear systems was introduced by El Jai et al. (1995) and consists in steering such a system to a desired state only in a subregion of the system evolution domain. Important developments and several works concerning linear parabolic systems were published (El Jai et al, 1995; Zerrik et al, 2004; Zerrik et al., 2000). Next, Zerrik and Larhrissi extended these results to linear hyperbolic systems and proposed approaches to the computation of the optimal control which allows us to reach a desired target in such a subregion (Zerrik et al, 2001; Zerrik et al, 2002). A natural direction is to extend theses results to semilinear distributed hyperbolic systems which constitute an original framework in regional analysis.

The objective of the present work is to study regional controllability for distributed semilinear hyperbolic systems using fixed point techniques combined with those established in (Zerrik et al, 2004) in the observation of semilinear parabolic systems in which interesting results were established and numerically performed. This is the aim of the paper, which is organized as follows:

We begin with some preliminaries. In the third section, we concentrate on the determination of a control achieving regional controllability when the system is asymptotically linear, i.e., for some $\alpha>0$ the nonlinear term $\mathcal{N}(s)$ satisfies

$$
\lim _{|s| \rightarrow+\infty} \frac{\mathcal{N}(s)}{s}=\alpha \quad \text { and } \mathcal{N}^{\prime} \in L^{\infty}(\mathbb{R}) .
$$

The analytical case is then considered using generalized inverse techniques. In all cases the control achieving a regional target is characterized via fixed point theorems and depends on the final time $T$, the subregion in question and the actuator location. In the last section, we provide a numerical approach which leads to an explicit formula for such a control with illustrations through numerical examples and simulations.

\section{Problem Statement}

Let $\Omega$ be a bounded open domain in $\mathbb{R}^{n}(n=1,2,3)$, with a regular boundary $\partial \Omega$. For $T>0$ we write $Q=\Omega \times] 0, T[, \Sigma=\partial \Omega \times] 0, T[$ and consider the following semilinear hyperbolic system:

$$
\begin{cases}\frac{\partial^{2} y}{\partial t^{2}}+\mathcal{A} y+\mathcal{N} y=\chi_{D} f u & \text { in } Q, \\ y(x, 0)=y_{0}(x), \frac{\partial y}{\partial t}(x, 0)=y_{1}(x) & \text { in } \Omega, \\ y(\xi, t)=0 & \text { on } \Sigma,\end{cases}
$$

where $\mathcal{A}$ is a second-order elliptic linear operator given by

$$
\mathcal{A}=-\sum_{i, j=1}^{n} \frac{\partial}{\partial x_{i}}\left(a_{i j} \frac{\partial}{\partial x_{j}}\right)
$$

with $a_{i j}(x)=a_{j i}(x) \in \mathcal{C}^{1}(\Omega)$, and there exists $\alpha_{1}>0$ such that

$$
\begin{aligned}
& \sum_{i, j=1}^{n} a_{i j} \xi_{i} \xi_{j} \geq \alpha_{1} \sum_{j=1}^{n}\left|\xi_{j}\right|^{2}, \\
& \forall \xi=\left(\xi_{1}, \xi_{2}, \ldots, \xi_{n}\right) \in \mathbb{R}^{n} .
\end{aligned}
$$

$\mathcal{N}$ is a nonlinear operator, $D \subset \Omega, f \in L^{2}(D)$, $u \in U=L^{2}(0, T)$ and $\left(y_{0}, y_{1}\right) \in H_{0}^{1}(\Omega) \times L^{2}(\Omega)$.

Let

$$
z_{u}=\left(y_{u}, \frac{\partial y_{u}}{\partial t}\right)
$$

denote the solution to (2) and assume that $z_{u}(T) \in$ $\mathcal{E}=\left(L^{2}(\Omega)\right)^{2}$. For $\omega$ being an open regular subset of $\Omega$ which has positive Lebesgue measure, consider the restriction operator

$$
\chi_{\omega}:\left\{\begin{array}{ccc}
L^{2}(\Omega) \times L^{2}(\Omega) & \rightarrow L^{2}(\omega) \times L^{2}(\omega), \\
\left(z_{1}, z_{2}\right) & \mapsto\left(\left.z_{1}\right|_{\omega},\left.z_{2}\right|_{\omega}\right),
\end{array}\right.
$$

where $\chi_{\omega}^{*}$ denotes its adjoint defined from $L^{2}(\omega) \times L^{2}(\omega)$ to $L^{2}(\Omega) \times L^{2}(\Omega)$ and given by

$$
\chi_{\omega}^{*}\left(z_{1}, z_{2}\right)(x)= \begin{cases}\left(z_{1}(x), z_{2}(x)\right), & x \in \omega, \\ (0,0), & x \in \Omega \backslash \omega .\end{cases}
$$

Definition 1. The system (2) is said to be w-exactly (resp. $\omega$-approximately) regionally controllable if for all $\left(z_{1}^{d}, z_{2}^{d}\right) \in L^{2}(\omega) \times L^{2}(\omega)$ (resp. for all $\varepsilon>0$ ) there exists $u \in U$ such that

$$
y_{u}(T)=z_{1}^{d}, \quad \frac{\partial y_{u}}{\partial t}(T)=z_{2}^{d} \text { in } \omega
$$


(resp. $\left.\left\|y_{u}(T)-z_{1}^{d}\right\|_{L^{2}(\omega)}+\left\|\frac{\partial y_{u}}{\partial t}(T)-z_{2}^{d}\right\|_{L^{2}(\omega)} \leq \varepsilon\right)$.

This definition generalizes the standard ones of exact and approximate controllabilities on the whole domain $\Omega$.

In the sequel, we consider the regional controllability problem for the system (2) excited by an internal zone actuator $(f, D)$ formulated as follows:

Problem 1. For $z^{d}=\left(z_{1}^{d}, z_{2}^{d}\right) \in L^{2}(\omega) \times L^{2}(\omega)$, find a control $u \in L^{2}(0, T)$ such that

$$
y_{u}(T)=z_{1}^{d}, \quad \frac{\partial y_{u}}{\partial t}(T)=z_{2}^{d} \text { in } \omega .
$$

Write $z=(y, \partial y / \partial t), A\left(z_{1}, z_{2}\right)=\left(z_{2}, \mathcal{A} z_{1}\right)$ for all $\left(z_{1}, z_{2}\right) \in D(A)=\left(H_{0}^{1}(\Omega) \cap H^{2}(\Omega)\right) \times L^{2}(\Omega), N z=$ $\left(0,-\mathcal{N} z_{1}\right), z_{0}=\left(y_{0}, y_{1}\right)$ and $B u=\left(0, \chi_{D} f u\right)$. The system (2) may be written as

$$
\begin{cases}\frac{\partial z}{\partial t}+A z=N z+B u & \text { in } Q \\ z(0)=z_{0} & \text { in } \Omega\end{cases}
$$

and its associated linear system is

$$
\begin{cases}\frac{\partial z}{\partial t}+A z=B u & \text { in } Q \\ z(0)=z_{0} & \text { in } \Omega .\end{cases}
$$

Assume that $(-A)$ generates a strongly continuous semigroup $S(t)_{(t \geq 0)}$ on the state space $\mathcal{E}$. Let $L(\cdot)$ and $G_{\omega}$ be the operators defined by

$$
\begin{aligned}
L(t) z(\cdot) & =\int_{0}^{t} S(t-s) z(s) \mathrm{d} s \\
\text { and } & \\
G_{\omega} u & =\chi_{\omega} L(T) B u .
\end{aligned}
$$

Consider now the function

$$
\begin{aligned}
& \Phi(z)(\cdot) \\
& =S(\cdot) z_{0}+L(\cdot) N z(\cdot) \\
& \quad+L(\cdot) B G_{\omega}^{\dagger}\left[z^{d}-\chi_{\omega} S(T) z_{0}-\chi_{\omega} L(T) N z(\cdot)\right]
\end{aligned}
$$

where $G_{\omega}^{\dagger}=\left(G_{\omega}^{*} G_{\omega}\right)^{-1} G_{\omega}^{*}$ is the generalized inverse of $G_{\omega}$.

Since $z^{*}(\cdot)$ is a fixed point of (6) such that $\left[z^{d}-\right.$ $\left.\chi_{\omega} S(T) z_{0}-\chi_{\omega} L(T) N z^{*}(\cdot)\right] \in \operatorname{Im} G_{\omega}$, it is easy to show that if (5) is $\omega$-approximately regionally controllable, then the control

$$
u^{*}=G_{\omega}^{\dagger}\left[z^{d}-\chi_{\omega} S(T) z_{0}-\chi_{\omega} L(T) N z^{*}(\cdot)\right]
$$

drives the system (2) to $z^{d}$ at time $T$.

\section{Proposed Approaches}

Here we shall study two important situations i.e., the case of asymptotically linear systems and the analytical one.

3.1. Asymptotically Linear Case. Here we deal with the problem (3) when the system (2) is assumed to satisfy (1). The approach we shall use is an extension of the Hilbert uniqueness method used to establish controllability in the linear case (Lions, 1988) and the semilinear case (Zuazua et al, 1990), and is developed in two steps.

Step 1. We consider the system (2) with $\mathcal{N} s=\alpha s$,

$$
\begin{cases}\frac{\partial^{2} y}{\partial t^{2}}+\mathcal{A} y+\alpha y=\chi_{D} f u & \text { in } Q, \\ y(x, 0)=y_{0}(x), \frac{\partial y}{\partial t}(x, 0)=y_{1}(x) & \text { in } \Omega, \\ y(\xi, t)=0 & \text { on } \Sigma,\end{cases}
$$

and let $G$ be the set

$$
\begin{aligned}
G= & \left\{\left(\phi_{1},-\phi_{0}\right) \in \mathcal{C}^{\infty}(\Omega) \times \mathcal{C}^{\infty}(\Omega)\right. \\
& \text { such that } \left.\phi_{0}=\phi_{1}=0 \text { on } \Omega \backslash \omega\right\} .
\end{aligned}
$$

For $\left(\phi_{1},-\phi_{0}\right) \in G$, the system

$$
\begin{cases}\frac{\partial^{2} \phi}{\partial t^{2}}+\mathcal{A} \phi+\alpha \phi=0 & \text { in } Q \\ \phi(x, T)=\phi_{0}(x), \frac{\partial \phi}{\partial t}(x, T)=\phi_{1}(x) & \text { in } \Omega \\ \phi(\xi, t)=0 & \text { on } \Sigma\end{cases}
$$

has a unique solution

$$
\phi \in C\left(0, T, H_{0}^{1}(\Omega)\right) \cap C^{1}\left(0, T, L^{2}(\Omega)\right),
$$

see (Lions, 1988).

In $G$ we define the following seminorm:

$$
\left\|\left(\phi_{1},-\phi_{0}\right)\right\|_{G}=\left(\int_{0}^{T}\langle\phi(t), f\rangle_{L^{2}(D)}^{2} \mathrm{~d} t\right)^{\frac{1}{2}}
$$

and consider the system

$$
\begin{cases}\frac{\partial^{2} \psi}{\partial t^{2}}+\mathcal{A} \psi+\alpha \psi=-\langle\phi, f\rangle_{L^{2}(D)} \chi_{D} f & \text { in } Q \\ \psi(x, 0)=y_{0}(x), \frac{\partial \psi}{\partial t}(x, 0)=y_{1}(x) & \text { in } \Omega \\ \psi(\xi, t)=0 & \text { on } \Sigma\end{cases}
$$

Here (11) has only one solution such that

$$
\left(\psi(T), \frac{\partial \psi}{\partial t}(T)\right) \in H_{0}^{1}(\Omega) \times L^{2}(\Omega),
$$

see (Lions, 1988). 
Let $M$ be the affine operator defined by

$$
M\left(\phi_{1},-\phi_{0}\right)=\mathcal{P}\left(\psi(T), \frac{\partial \psi}{\partial t}(T)\right)
$$

with $\mathcal{P}=\chi_{\omega}^{*} \chi_{\omega}$.

But

$\left(\psi(T), \frac{\partial \psi}{\partial t}(T)\right)=\left(\psi_{0}(T), \frac{\partial \psi_{0}}{\partial t}(T)\right)+\left(\psi_{1}(T), \frac{\partial \psi_{1}}{\partial t}(T)\right)$,

where $\psi_{0}$ and $\psi_{1}$ are solutions of the systems

$$
\begin{cases}\frac{\partial^{2} \psi_{0}}{\partial t^{2}}+\mathcal{A} \psi_{0}+\alpha \psi_{0}=0 & \text { in } Q, \\ \psi_{0}(x, 0)=y_{0}(x), \frac{\partial \psi_{0}}{\partial t}(x, 0)=y_{1}(x) & \text { in } \Omega, \\ \psi_{0}(\xi, t)=0 & \text { on } \Sigma\end{cases}
$$

and

$$
\begin{cases}\frac{\partial^{2} \psi_{1}}{\partial t^{2}}+\mathcal{A} \psi_{1}+\alpha \psi_{1}=-\langle\phi, f\rangle_{L^{2}(D)} \chi_{D} f & \text { in } Q \\ \psi_{1}(x, 0)=0, \frac{\partial \psi_{1}}{\partial t}(x, 0)=0 & \text { in } \Omega \\ \psi_{1}(\xi, t)=0 & \text { on } \Sigma .\end{cases}
$$

We consider the operator

$$
\Lambda\left(\phi_{1},-\phi_{0}\right)=\mathcal{P}\left(\psi_{1}(T), \frac{\partial \psi_{1}}{\partial t}(T)\right) .
$$

$\Lambda$ is a symmetric and bounded operator (Zuazua et al, 1990), then the regional controllability problem for the system (8) reduces to solving the equation

$$
\Lambda\left(\phi_{1},-\phi_{0}\right)=-\mathcal{P}\left(\psi_{0}(T), \frac{\partial \psi_{0}}{\partial t}(T)\right)+\chi_{\omega}^{*}\left(z_{1}^{d}, z_{2}^{d}\right)
$$

Theorem 1. If (8) is $\omega$-approximately regionally controllable, then (15) has a unique solution $\left(\phi_{1},-\phi_{0}\right)$ and $u^{*}(t)=-\langle\phi(t), f\rangle_{L^{2}(D)}$ drives the linear system (8) to $z^{d}$ in $\omega$ at time $T$, where $\phi$ is a solution of the system (9).

Proof. Since (8) is approximately controllable, (10) is a norm and $\Lambda$ is an isomorphism from $\widehat{G}$ into $\widehat{G}^{*}$, where $\widehat{G}$ is the completion of $G$ with respect to the norm (10) and $\widehat{G}^{*}$ is its dual. (The details of the proof are given in (El Jai et al, 1995).)

Step 2. Let $\mathcal{N}$ satisfy (1). Then the solution of the system (2) can be written as

$$
y=\psi_{0}+\psi_{1}+\psi_{2},
$$

where $\psi_{0}$ and $\psi_{1}$ are respectively solutions of the systems (12) and (13) which satisfy (Lions, 1988)

$$
\psi_{0}, \psi_{1} \in C\left(0, T, H_{0}^{1}(\Omega)\right) \cap C^{1}\left(0, T, L^{2}(\Omega)\right) .
$$

Moreover, there exists a positive constant $\beta_{1}$ such that

$$
\begin{aligned}
\left\|\psi_{0}\right\|_{L^{\infty}\left(0, T, H_{0}^{1}(\Omega)\right)}+ & \left\|\frac{\partial \psi_{0}}{\partial t}\right\|_{L^{\infty}\left(0, T, L^{2}(\Omega)\right)} \\
& \leq \beta_{1}\left\|\left(y_{0}, y_{1}\right)\right\|_{H_{0}^{1}(\Omega) \times L^{2}(\Omega)}
\end{aligned}
$$

$$
\begin{aligned}
\left\|\psi_{1}\right\|_{L^{\infty}\left(0, T, H_{0}^{1}(\Omega)\right)}+ & \left\|\frac{\partial \psi_{1}}{\partial t}\right\|_{L^{\infty}\left(0, T, L^{2}(\Omega)\right)} \\
& \leq \beta_{1}\left\|\left(\phi_{0}, \phi_{1}\right)\right\|_{H_{0}^{1}(\Omega) \times L^{2}(\Omega)},
\end{aligned}
$$

and $\psi_{2}$ is a solution of the system

$$
\left\{\begin{array}{cc}
\frac{\partial^{2} \psi_{2}}{\partial t^{2}}+\mathcal{A} \psi_{2}+\mathcal{N}\left(\psi_{0}+\psi_{1}+\psi_{2}\right) & \\
=\alpha\left(\psi_{0}+\psi_{1}\right) & \text { in } Q, \\
\psi_{2}(x, 0)=0, \frac{\partial \psi_{2}}{\partial t}(x, 0)=0 & \text { in } \Omega, \\
\psi_{2}(\xi, t)=0 & \text { on } \Sigma,
\end{array}\right.
$$

and since $\mathcal{N}^{\prime} \in L^{\infty}(\mathbb{R})$, the mapping $\psi \longrightarrow \mathcal{N}\left(\psi_{0}(t)+\right.$ $\left.\psi_{1}(t)+\psi(t)\right)$ is Lipschitz continuous from $L^{2}(\Omega) \rightarrow$ $L^{2}(\Omega)$ a.e. in $[0, T]$. Then (18) has a unique solution (Lions, 1988):

$$
\psi_{2} \in C\left(0, T, H_{0}^{1}(\Omega)\right) \cap C^{1}\left(0, T, L^{2}(\Omega)\right) .
$$

Let us now define the nonlinear operator

$$
\begin{aligned}
& \mu\left(\phi_{1},-\phi_{0}\right) \\
& \quad=\mathcal{P}\left(\psi_{1}(T), \frac{\partial \psi_{1}}{\partial t}(T)\right)+\mathcal{P}\left(\psi_{2}(T), \frac{\partial \psi_{2}}{\partial t}(T)\right) .
\end{aligned}
$$

Then the problem of regional controllability of (2) reduces to solving the equation

$$
\mu\left(\phi_{1},-\phi_{0}\right)=\chi_{\omega}^{*}\left(z_{1}^{d}, z_{2}^{d}\right)-\mathcal{P}\left(\psi_{0}(T), \frac{\partial \psi_{0}}{\partial t}(T)\right) .
$$

By (14), Eqn. (20) is equivalent to

$$
\begin{aligned}
\Lambda\left(\phi_{1},-\phi_{0}\right)= & \chi_{\omega}^{*}\left(z_{1}^{d}, z_{2}^{d}\right)-\mathcal{P}\left(\psi_{2}(T), \frac{\partial \psi_{2}}{\partial t}(T)\right) \\
& -\mathcal{P}\left(\psi_{0}(T), \frac{\partial \psi_{0}}{\partial t}(T)\right) .
\end{aligned}
$$

For a large constant $\beta_{2}>0$, consider the set

$$
\begin{aligned}
& \mathcal{G}=\left\{\left(\phi_{1},-\phi_{0}\right) \in G\right. \text { such that } \\
&\left.\left\|\left(\phi_{0}, \phi_{1}\right)\right\|_{H_{0}^{1}(\Omega) \times L^{2}(\Omega)} \leq \beta_{2}\left\|\left(\phi_{1},-\phi_{0}\right)\right\|_{G}\right\} .
\end{aligned}
$$

Then a solution of (20) is a fixed point of the nonlinear operator

$$
\begin{aligned}
\tilde{\mu}\left(\phi_{1},-\phi_{0}\right)= & \Lambda^{-1} \chi_{\omega}^{*}\left(z_{1}^{d}, z_{2}^{d}\right)-\Lambda^{-1} \mathcal{K}_{\omega}\left(\phi_{1},-\phi_{0}\right) \\
& -\Lambda^{-1} \mathcal{P}\left(\psi_{0}(T), \frac{\partial \psi_{0}}{\partial t}(T)\right)
\end{aligned}
$$


where $\mathcal{K}_{\omega}$ is the operator defined by

$$
\mathcal{K}_{\omega}:\left\{\begin{array}{cl}
\mathcal{G} & \rightarrow \widehat{G}^{*} \\
\left(\phi_{1},-\phi_{0}\right) & \mapsto \mathcal{P}\left(\psi_{2}(T), \frac{\partial \psi_{2}}{\partial t}(T)\right)
\end{array}\right.
$$

Theorem 2. If the system (8) is $\omega$-approximately regionally controllable, then (22) has a unique fixed point $\left(\phi_{1},-\phi_{0}\right)$ and the control $u^{*}(t)=-\langle\phi(t), f\rangle_{L^{2}(D)}$ drives the system (2) to $z^{d}$ in $\omega$ at time $T$, where $\phi$ is a solution of the system (9).

Proof. We have $\psi_{2} \in C\left(0, T, H_{0}^{1}(\Omega)\right) \cap C^{1}\left(0, T, L^{2}(\Omega)\right)$ and there exists $\beta_{3}>0$ such that for all $t>0$

$$
\begin{aligned}
& \left\|\mathcal{P}\left(\frac{\partial \psi_{2}}{\partial t}(t),-\psi_{2}(t)\right)\right\|_{\widehat{G}^{*}} \\
& \quad \leq \beta_{3}\left[\left\|\psi_{2}(t)\right\|_{H_{0}^{1}(\Omega)}+\left\|\frac{\partial \psi_{2}(t)}{\partial t}\right\|_{L^{2}(\Omega)}\right]
\end{aligned}
$$

Then $\mathcal{P}\left(\frac{\partial \psi_{2}}{\partial t},-\psi_{2}\right) \in C\left(0, T, \widehat{G}^{*}\right)$.

There exist $\varepsilon>0$ and $\beta_{4}>0$ (Lions, 1988) such that

$$
\begin{aligned}
\| \psi_{2} & \left\|_{L^{\infty}\left(0, T, H_{0}^{1}(\Omega)\right)}+\right\| \frac{\partial \psi_{2}}{\partial t} \|_{L^{\infty}\left(0, T, L^{2}(\Omega)\right)} \\
\leq & \varepsilon\left(\left\|\left(y_{0}, y_{1}\right)\right\|_{H_{0}^{1}(\Omega) \times L^{2}(\Omega)}+\left\|\left(\phi_{0}, \phi_{1}\right)\right\|_{H_{0}^{1}(\Omega) \times L^{2}(\Omega)}\right) \\
& +\beta_{4} .
\end{aligned}
$$

Moreover, since $\left(\phi_{1},-\phi_{0}\right) \in \mathcal{G}$, for all $t>0$ we have

$$
\begin{aligned}
& \left\|\mathcal{P}\left(\frac{\partial \psi_{2}}{\partial t}(t),-\psi_{2}(t)\right)\right\|_{\widehat{G}^{*}} \\
& \leq \varepsilon\left[\left\|\left(y_{0}, y_{1}\right)\right\|_{H_{0}^{1}(\Omega) \times L^{2}(\Omega)}+\beta_{2}\left\|\left(\phi_{1},-\phi_{0}\right)\right\|_{G}+\frac{\beta_{4}}{\varepsilon}\right] .
\end{aligned}
$$

Applying (24) with $\varepsilon=\left[2 \beta_{2}\left\|\Lambda^{-1}\right\|_{\mathcal{L}\left(G^{*}, G\right)}\right]^{-1}$, for some constant $\beta_{5}>0$ we have

$$
\begin{aligned}
& \left\|\tilde{\mu}\left(\phi_{1},-\phi_{0}\right)\right\|_{G} \\
& \leq\left\|\Lambda^{-1} \mathcal{K}_{\omega}\left(\phi_{1},-\phi_{0}\right)\right\|_{G} \\
& \quad+\left\|\Lambda^{-1} \chi_{\omega}^{*}\left(z_{1}^{d}, z_{2}^{d}\right)-\Lambda^{-1} \mathcal{P}\left(\psi_{0}(T), \frac{\partial \psi_{0}}{\partial t}(T)\right)\right\|_{G} \\
& \leq \frac{1}{2}\left\|\left(\phi_{1},-\phi_{0}\right)\right\|_{G}+\beta_{5} .
\end{aligned}
$$

Moreover, from (23) and (24), $\mathcal{K}_{\omega}$ is a compact operator. Then $\tilde{\mu}$ is also compact and there exists $M \geq 2 \beta_{5}$ such that

$$
\left\|\tilde{\mu}\left(\phi_{1},-\phi_{0}\right)\right\|_{G} \leq M
$$

for any $\left(\phi_{1},-\phi_{0}\right) \in G$ satisfying

$$
\left\|\left(\phi_{1},-\phi_{0}\right)\right\|_{G} \leq M \text {. }
$$

Hence, by applying Schauder's fixed-point theorem, (Zeidler, 1990) the operator (22) has at least one fixed point and the proof is completed.

\section{Remark 1.}

1. The approach used here is a natural generalization of the one developed for the linear case. Indeed, when $\mathcal{N} s=\alpha s$, the operator $\mu$ coincides with the isomorphism $\Lambda$ given by (14).

2. The problem (3) can be solved by similar techniques when the system is excited by a boundary actuator.

3.2. Analytical Case. In the following, we consider the problem (3) for the system (4) with $z_{0}=0$ and assume that $(-A)$ generates an analytic semi-group $S(t)_{(t \geq 0)}$ on the state space $\mathcal{E}$. Moreover, let $A_{1}=A+a I$, where $a$ is a real such that $\operatorname{Re} \sigma\left(A_{1}\right)>\delta>0$ while $\operatorname{Re} \sigma\left(A_{1}\right)$ indicates the real part of the spectrum of $A_{1}$. Then for $0 \leq \alpha<1, \mathcal{E}^{\alpha}=D\left(A_{1}^{\alpha}\right)$ defines a dense Banach space on $\mathcal{E}$ endowed with the graph norm

$$
\|\cdot\|_{\mathcal{E}^{\alpha}}=\left\|A_{1}^{\alpha}(\cdot)\right\|_{\mathcal{E}}
$$

and $\|S(t)\|_{\mathcal{L}\left(\mathcal{E}, \mathcal{E}^{\alpha}\right)}=c \cdot t^{-\alpha} \exp (a-\delta) t=g_{1}(t)$ (Zeidler, 1990).

Assume that $g_{1} \in L^{q}(0, T), q \geq 1$, and let $r, s \geq 1$ be such that

$$
\frac{1}{q}=1+\frac{1}{r}-\frac{1}{s}
$$

and that $N$ is well defined from $L^{r}\left(0, T ; \mathcal{E}^{\alpha}\right) \rightarrow$ $L^{s}(0, T ; \mathcal{E})$ satisfying

$$
\left\{\begin{array}{l}
N(0)=0 \\
\|N x-N y\|_{L^{s}(0, T ; \mathcal{E})} \\
\quad \leq k(\|x\|,\|y\|)\|x-y\|_{L^{r}\left(0, T ; \mathcal{E}^{\alpha}\right)}, \\
\quad \forall x, y \in L^{r}\left(0, T ; \mathcal{E}^{\alpha}\right) \\
\text { with } \quad k: \mathbb{R}^{+} \times \mathbb{R}^{+} \longrightarrow \mathbb{R}^{+} \\
\text {such that } \lim _{\theta_{1}, \theta_{2} \rightarrow 0} k\left(\theta_{1}, \theta_{2}\right)=0 .
\end{array}\right.
$$

These hypotheses are fulfilled by many important classes of semilinear hyperbolic systems. Various examples are given and discussed in (Henry, 1981; Kassara et al, 1983).

Consider now the functions

$$
\tilde{\Phi}(z, u)=L(\cdot) N z+L(\cdot) B u
$$

and

$$
\tilde{\psi}_{\omega}\left(z^{d}, u\right)=G_{\omega}^{\dagger}\left(z^{d}-\chi_{\omega} L(T) N z_{u}\right) .
$$

In the following, $\operatorname{Im} G_{\omega}$ is endowed with the seminorm

$$
\left\|z^{d}\right\|_{\operatorname{Im} G_{\omega}}=\left\|G_{\omega}^{\dagger} z^{d}\right\|_{L^{2}(0, T)} .
$$

Theorem 3. Assume that the system (5) is $\omega$ approximately regionally controllable, the hypothesis (25) holds and

$$
\|L(\cdot) B u\|_{L^{r}\left(0, T, \mathcal{E}^{\alpha}\right)} \leq \beta\|u\|_{L^{2}(0, T)},
$$




$$
\left\|\chi_{\omega} S(\cdot)\right\|_{\mathcal{L}\left(\mathcal{E}, \operatorname{Im} G_{\omega}\right)}=g_{2} \in L^{d}(0, T)
$$

such that

$$
\frac{1}{d}+\frac{1}{s}=1
$$

\section{Then}

1. There exist $m>0$ and $\rho>0$ such that for any $z^{d} \in B(0, \rho) \subset \operatorname{Im} G_{\omega}$ there exists $u^{*} \in B(0, m)$ being the unique solution to Problem 1.

2. The mapping $z^{d} \mapsto u^{*}\left(z^{d}\right)$ from $B(0, \rho) \rightarrow$ $L^{2}(0, T)$ is Lipschitz.

Proof. 1. Since the system (5) is $\omega$-approximately controllable, $G_{\omega}^{\dagger}$ is injective and consequently (28) is a norm.

2. We have

$$
\lim _{\theta_{1}, \theta_{2} \rightarrow 0} k\left(\theta_{1}, \theta_{2}\right)=0 .
$$

Hence there exists $\gamma>0$ such that

$$
C_{1}:=\left\|g_{2}\right\|_{L^{q}(0, T)} \sup _{\theta_{1}, \theta_{2}<\gamma} k\left(\theta_{1}, \theta_{2}\right)<C_{2}
$$

and

$$
\begin{aligned}
C_{2}:= & \left(\beta\left\|g_{2}\right\|_{L^{d}(0, T)}+\left\|g_{1}\right\|_{L^{q}(0, T)}\right) \\
& \times \sup _{\theta_{1}, \theta_{2}<\gamma} k\left(\theta_{1}, \theta_{2}\right)<1 .
\end{aligned}
$$

But it is well known (Kassara et al, 1983) that there exists

$$
m:=\frac{\gamma}{\beta}\left(1-\left\|g_{1}\right\|_{L^{q}(0, T)} \sup _{\theta \leq \gamma} k(\theta, 0)\right)
$$

such that for all $u \in B(0, m), \tilde{\Phi}(\cdot, u)$ has only one fixed point $z \in B(0, \gamma) \subset L^{r}\left(0, T, \mathcal{E}^{\alpha}\right)$ being a solution of (4) and the mapping $u \mapsto z_{u}$ is Lipschitz with constant $\beta /\left(1-C_{1}\right)$. Then, for $z^{d} \in \operatorname{Im} G_{\omega}$ we have

$$
\begin{aligned}
& \left\|\tilde{\psi}_{\omega}\left(z^{d}, u\right)-\tilde{\psi}_{\omega}\left(z^{d}, v\right)\right\|_{L^{2}(0, T)} \\
& \quad=\left\|G_{\omega}^{\dagger} \chi_{\omega} L(T)\left(N z_{v}-N z_{u}\right)\right\|_{L^{2}(0, T)} \\
& \quad=\left\|\chi_{\omega} L(T)\left(N z_{v}-N z_{u}\right)\right\|_{\operatorname{Im} G_{\omega}} \\
& \quad \leq\left\|g_{2}\right\|_{L^{d}(0, T)}\left\|N z_{v}-N z_{u}\right\|_{L^{s}(0, T, \mathcal{E})} \\
& \quad \leq \frac{\beta}{1-C_{1}}\left\|g_{2}\right\|_{L^{d}(0, T)} \sup _{\theta_{1}, \theta_{2}<\gamma} k\left(\theta_{1}, \theta_{2}\right)\|u-v\| .
\end{aligned}
$$

Consequently,

$$
\left\|\tilde{\psi}_{\omega}\left(z^{d}, u\right)-\tilde{\psi}_{\omega}\left(z^{d}, v\right)\right\|_{L^{2}(0, T)} \leq C_{3}\|u-v\|,
$$

where

$$
C_{3}:=\frac{\beta}{1-C_{1}}\left\|g_{2}\right\|_{L^{d}(0, T)} \sup _{\theta_{1}, \theta_{2}<\gamma} k\left(\theta_{1}, \theta_{2}\right)<1,
$$

which shows that $\tilde{\psi}_{\omega}$ is a contraction.

Moreover,

$$
\begin{aligned}
\left\|\tilde{\psi}_{\omega}\left(z^{d}, u\right)\right\| & =\left\|z^{d}-\chi_{\omega} L(T) N z_{u}\right\| \\
& \leq\left\|z^{d}\right\|+\left\|\chi_{\omega} L(T) N z_{u}\right\| \\
& \leq\left\|z^{d}\right\|+\left\|g_{2}\right\|_{L^{d}(0, T)} k\left(\left\|z_{u}\right\|, 0\right)\left\|z_{u}\right\| \\
& \leq\left\|z^{d}\right\|+\left\|g_{2}\right\|_{L^{d}(0, T)} \sup _{\theta \leq \gamma} k(\theta, 0) \gamma .
\end{aligned}
$$

Thus, if $u \in B(0, m)$ and

$$
\left\|z^{d}\right\| \leq m-\left\|g_{2}\right\|_{L^{d}(0, T)} \sup _{\theta \leq \gamma} k(\theta, 0) \gamma,
$$

then $\tilde{\psi}_{\omega}\left(z^{d}, u\right) \in B(0, m)$.

From (31) we obtain

$\left\|z^{d}\right\|$

$$
\begin{aligned}
& \leq \frac{\gamma}{\beta}\left(1-\left(\left\|g_{1}\right\|_{L^{q}(0, T)}+\beta\left\|g_{2}\right\|_{L^{d}(0, T)}\right) \sup _{\theta \leq \gamma} k(\theta, 0)\right) \\
& =: \rho .
\end{aligned}
$$

Consequently, if $z^{d} \in B(0, \rho) \subset \operatorname{Im} G_{\omega}$, then $\tilde{\psi}_{\omega}\left(z^{d}, \cdot\right)$ has a unique fixed point in $B(0, m)$ being a solution to the problem (3).

3. For $z^{d}, y^{d} \in B(0, \rho)$ we have

$$
\begin{aligned}
u^{*}\left(z^{d}\right)- & u^{*}\left(y^{d}\right) \\
= & \tilde{\psi}_{\omega}\left(z^{d}, u^{*}\left(z^{d}\right)\right)-\tilde{\psi}_{\omega}\left(y^{d}, u^{*}\left(y^{d}\right)\right) \\
= & \tilde{\psi}_{\omega}\left(z^{d}, u^{*}\left(z^{d}\right)\right)-\tilde{\psi}_{\omega}\left(z^{d}, u^{*}\left(y^{d}\right)\right) \\
& +\tilde{\psi}_{\omega}\left(z^{d}, u^{*}\left(y^{d}\right)\right)-\tilde{\psi}_{\omega}\left(y^{d}, u^{*}\left(y^{d}\right)\right) .
\end{aligned}
$$

But

$$
\begin{aligned}
& \left\|\tilde{\psi}_{\omega}\left(z^{d}, u^{*}\left(z^{d}\right)\right)-\tilde{\psi}_{\omega}\left(z^{d}, u^{*}\left(y^{d}\right)\right)\right\| \\
& \leq C_{3}\left\|u^{*}\left(z^{d}\right)-u^{*}\left(y^{d}\right)\right\|, \\
& \left\|\tilde{\psi}_{\omega}\left(z^{d}, u^{*}\left(y^{d}\right)\right)-\tilde{\psi}_{\omega}\left(y^{d}, u^{*}\left(y^{d}\right)\right)\right\|=\left\|z^{d}-y^{d}\right\| .
\end{aligned}
$$

Hence

$$
\left\|u^{*}\left(z^{d}\right)-u^{*}\left(y^{d}\right)\right\| \leq \frac{1}{1-C_{3}}\left\|z^{d}-y^{d}\right\|,
$$

which shows that the mapping $z^{d} \longrightarrow u^{*}\left(z^{d}\right)$ from $B(0, \rho) \rightarrow L^{2}(0, T)$ is Lipshitz.

Proposition 1. The sequence of controls

$$
\left\{\begin{array}{l}
u_{n+1}=G_{\omega}^{\dagger}\left(z^{d}-\chi_{\omega} L(T) N z_{u_{n}}\right), \\
u_{0}=0
\end{array}\right.
$$

converges in $L^{2}(0, T)$ to $u^{*}$, a solution to Problem 1.

Proof. The proof proceeds using (32) and (28). 


\section{Numerical Approach}

Here we outline a numerical approach that leads to the computation of the control solution to Problem 1 when the system (4) is analytic. From (34) the control satisfies

$$
G_{\omega}^{\dagger}\left(z^{d}-\chi_{\omega} z_{u_{n}}\right)=u_{n+1}-u_{n}
$$

and the problem reduces to computing $G_{\omega}^{\dagger}$.

Since the system (5) is $\omega$-approximately regionally controllable, $\left(G_{\omega}^{*} G_{\omega}\right)$ is invertible and the operator $G_{\omega}^{\dagger}=$ $\left(G_{\omega}^{*} G_{\omega}\right)^{-1} G_{\omega}^{*}$ is well defined and may be written as

$$
G_{\omega}^{\dagger} y=\sum_{i \geq 1} \tilde{z}_{i} w_{i}(t)
$$

where

$$
w_{i}(t)=\left(\frac{2}{T}\right)^{\frac{1}{2}} \sin \left(\frac{i \pi t}{T}\right)
$$

with $i \geq 1$, and the problem is to calculate the component $\tilde{z}_{i}$.

If $\Phi_{n}$ are the eigenfunctions of $A$, then the operator $G_{\omega}: L^{2}(0, T) \rightarrow L^{2}(\omega) \times L^{2}(\omega)$ may be written as

$$
\begin{aligned}
& G_{\omega} u \\
& =\left[\begin{array}{l}
\left.\sum_{n \geq 1} \frac{1}{n \pi}\left\langle\chi_{D} f, \Phi_{n}\right\rangle\langle u(\cdot), \sin n \pi(T-\cdot)\rangle \Phi_{n}\right|_{\omega} \\
\left.\sum_{n \geq 1}\left\langle\chi_{D} f, \Phi_{n}\right\rangle\langle u(\cdot), \cos n \pi(T-\cdot)\rangle \Phi_{n}\right|_{\omega}
\end{array}\right] .
\end{aligned}
$$

Hence, for all $v \in L^{2}(0, T)$ and $z=\left(z_{1}, z_{2}\right) \in L^{2}(\omega) \times$ $L^{2}(\omega)$ we have

$$
\begin{aligned}
& \left\langle G_{\omega} v,\left(z_{1}, z_{2}\right)\right\rangle \\
& =\left\langle\sum_{n \geq 1} \frac{1}{n \pi}\left\langle\chi_{D} f, \Phi_{n}\right\rangle\left\langle\Phi_{n}, z_{1}\right\rangle_{\omega}\langle\sin n \pi(T-\cdot), v(\cdot)\rangle\right. \\
& +\sum_{n \geq 1}\left\langle\chi_{D} f, \Phi_{n}\right\rangle\left\langle\Phi_{n}, z_{2}\right\rangle_{\omega}\langle\cos n \pi(T-\cdot), v(\cdot)\rangle,
\end{aligned}
$$

and $G_{\omega}^{*}: L^{2}(\omega) \times L^{2}(\omega) \rightarrow L^{2}(0, T)$ is given by

$$
\begin{aligned}
& G_{\omega}^{*}\left(z_{1}, z_{2}\right) \\
& =\sum_{m \geq 1} \frac{1}{m \pi}\left\langle\chi_{D} f, \Phi_{m}\right\rangle\left\langle\Phi_{m}, \tilde{\chi}_{\omega}^{*} z_{1}\right\rangle_{\Omega} \sin m \pi(T-\cdot) \\
& +\sum_{m \geq 1}\left\langle\chi_{D} f, \Phi_{m}\right\rangle\left\langle\Phi_{m}, \tilde{\chi}_{\omega}^{*} z_{2}\right\rangle_{\Omega} \cos m \pi(T-\cdot) .
\end{aligned}
$$

Then, for $j \geq 1$,

$$
\begin{aligned}
& \left\langle G_{\omega}^{*}\left(z^{d}-\chi_{\omega}\left(y_{u}(T), \frac{\partial y_{u}}{\partial t}(T)\right)\right), w_{j}\right\rangle \\
& =\sum_{m \geq 1} \frac{\mathcal{R}(m)}{m \pi}\left[\left\langle\Phi_{m}, z_{1}^{d}\right\rangle_{\omega}-\left\langle\Phi_{m}, y_{u}(T)\right\rangle_{\omega}\right] \Theta(j, m) \\
& \quad+\sum_{m \geq 1} \mathcal{R}(m)\left[\left\langle\Phi_{m}, z_{2}^{d}\right\rangle_{\omega}-\left\langle\Phi_{m}, \frac{\partial y_{u}}{\partial t}(T)\right\rangle_{\omega}\right] \mathcal{D}(j, m) \\
& =: \mathcal{Y}_{j}
\end{aligned}
$$

and

$$
\begin{aligned}
& \left\langle G_{\omega}^{*} G_{\omega} w_{i}, w_{j}\right\rangle \\
& =\sum_{m \geq 1} \frac{1}{m \pi} \mathcal{R}(m) \sum_{n \geq 1}\left[\frac{\Pi(m, n) \mathcal{R}(n) \Theta(i, n)}{n \pi}\right] \Theta(j, m) \\
& \quad+\sum_{m \geq 1} \mathcal{R}(m) \sum_{n \geq 1}[\Pi(m, n) \mathcal{R}(n) \mathcal{D}(i, n)] \mathcal{D}(j, m) \\
& =: \quad \tilde{A}_{i j},
\end{aligned}
$$

where

$$
\left\{\begin{array}{l}
\mathcal{R}(m)=\left\langle f, \Phi_{m}\right\rangle_{D}, \quad \Theta(i, n)=\left\langle w_{i}, \sin n \pi(T-\cdot)\right\rangle, \\
\Pi(n, m)=\left\langle\Phi_{n}, \Phi_{m}\right\rangle_{\omega}, \mathcal{D}(i, n)=\left\langle w_{i}, \cos n \pi(T-\cdot)\right\rangle,
\end{array}\right.
$$

and $\tilde{z}_{j}$ are solutions of the system

$$
\sum_{j \geq 1} \tilde{A_{i j}} \mathcal{Z}_{j}=\mathcal{Y}_{i}, \quad i=1,2, \ldots
$$

where $\left(\tilde{A}_{i j}\right)$ are given by 39 and $\mathcal{Y}_{j}$ by 38 . From 35 the control $u_{n}^{*}$ is approximated by

$$
u_{n+1}^{*} \simeq \sum_{i=1}^{M} \tilde{z}_{i} w_{i}+u_{n}^{*}, \quad M \in \mathbb{N}^{*} .
$$

Let $z_{u_{n}^{*}}$ be the solution of the system (4) excited by $u_{n}^{*}$ and

$$
\mathcal{Y}_{j}=\left(\left\langle G_{\omega}^{*}\left(z^{d}-\chi_{\omega} z_{u_{n+1}^{*}}(T)\right), w_{j}\right\rangle\right), \quad j \leq M .
$$

Accordingly, the algorithm can be implemented as follows:

Step 1: Let $\left(z_{1}^{d}, z_{2}^{d}\right)$, the region $\omega$ and the actuator location $D$. Choose the truncation order $M$.

\section{Step 2: Repeat}

- Solve the system 40).

- Computation of the control $u_{n+1}^{*}$ using (41).

- Solve the system (4).

- Solve (42) to obtain $\mathcal{Y}_{j}$.

Until $\left(\left\|z^{d}-\chi_{\omega} z_{u_{n+1}^{*}}(T)\right\|<\varepsilon\right)$.

Step 3: Let $\left.z^{*}(T)\right|_{\omega}=\chi_{\omega} z_{u_{n+1}^{*}}(T)$, which approaches the desired state in $\omega$. 


\section{Simulations Results}

In this part, we provide a numerical example and simulation results related to the choice of the subregion, the desired state and the actuator location.

Example 1. Consider the one-dimensional system excited by a zone actuator located in $D$ :

$$
\begin{cases}\frac{\partial^{2} y(x, t)}{\partial t_{m}^{2}}-\frac{\partial^{2} y(x, t)}{\partial x^{2}} & \\ \quad+\sum_{i=1}^{m}\left|\left\langle y(t), w_{i}\right\rangle\right|\left\langle y(t), w_{i}\right\rangle w_{i}(x) & \text { in }] 0,1[\times] 0, T[, \\ +\chi_{D}(x) \mathbb{1}(x) u(t)=0 & \text { in }] 0,1[, \\ y(x, 0)=0, \quad \frac{\partial y}{\partial t}(x, 0)=0 & \text { on }] 0, T[,\end{cases}
$$

where $w_{i}(x)=\sqrt{2} \sin (i \pi x), i \in \mathbb{N}^{*}$ and $\left.D=\right] 0,2 ; 0,4[$.

Let $z_{1}^{d}=A_{1} \sin (\pi x)$ and $z_{2}^{d}=A_{1}\left(1+B_{1}\right) \sin (\pi x)$ be the desired state in $\omega=] 0.4,0.8[$. For numerical considerations, $A_{1}$ and $B_{1}$ are chosen in order to produce a desired state with a reasonable amplitude.

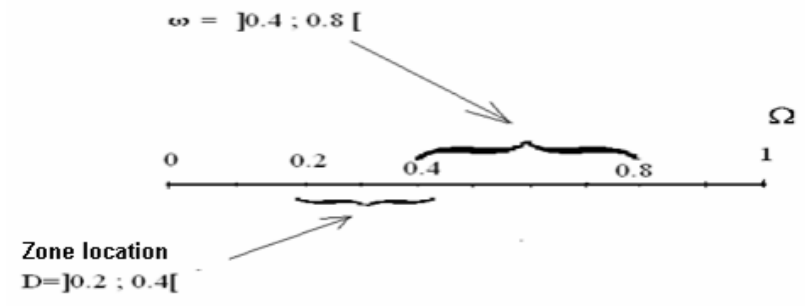

Fig. 1. Actuator location and the subregion.

By taking $T=1$ and applying the previous algorithm, the desired state is obtained with error

$\left\|y_{u^{\star}}(T)-z_{1}^{d}\right\|_{L^{2}(\omega)}+\left\|\frac{\partial y_{u^{\star}}}{\partial t}(T)-z_{2}^{d}\right\|_{L^{2}(\omega)}=4.2 \times 10^{-4}$ and cost

$$
\left\|u^{\star}\right\|_{L^{2}(0, T)}=1.38 \times 10^{-6} .
$$

Example 2. Now consider the one-dimensional system excited by a pointwise actuator located at $b$ :

$$
\begin{cases}\frac{\partial^{2} y(x, t)}{\partial t^{2}}-\frac{\partial^{2} y(x, t)}{\partial x^{2}} & \\ =-|y(x, t)| y(x, t)+\delta(x-b) u(t) \\ y(x, 0)=0, \quad \frac{\partial y}{\partial t}(x, 0)=0 & \text { in }] 0,1[\times] 0, \mathrm{~T}[, \\ y(0, t)=y(1, t)=0 & \text { on }] 0, \mathrm{~T}[.\end{cases}
$$

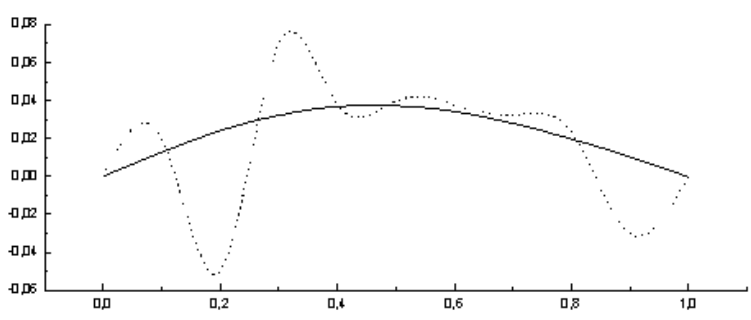

Fig. 2. Desired (dashed line) and final (solid line) position in $\omega$.

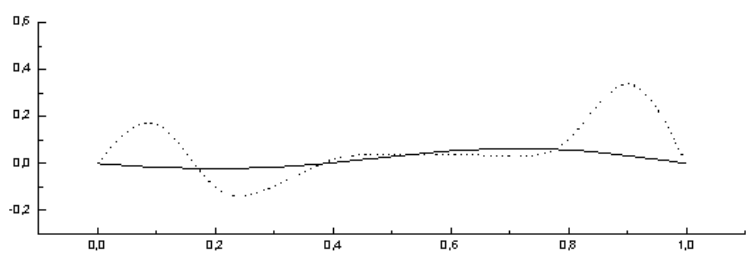

Fig. 3. Desired (dashed line) and final (solid line) speed in $\omega$.

Since the initial conditions are regular enough, we have a regular system state (Brezis, 1993), so with a pointwise actuator we obtain a similar result as in the zone case.

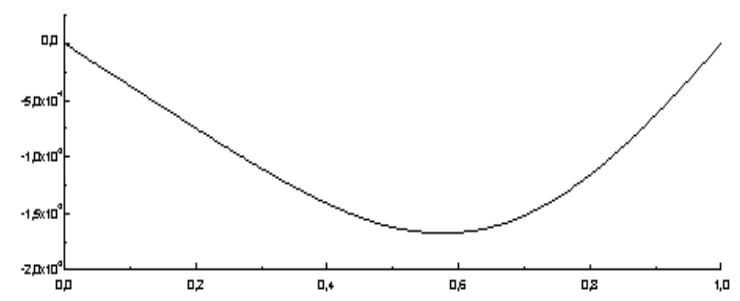

Fig. 4. Evolution of the control function on the time interval $[0, T]$.

Let $z_{1}^{d}=A_{1} \sin (\pi x), z_{2}^{d}=A_{1}\left(1+B_{1}\right) \sin (\pi x)$ be the desired state in $\omega=] 0.4,0.7[$ and $b=0.2$. For numerical considerations, $A_{1}$ and $B_{1}$ are chosen in order to yield a desired state with a reasonable amplitude.

Taking $T=1$ and applying the previous algorithm, the desired state is obtained with error

$\left\|y_{u^{\star}}(T)-z_{1}^{d}\right\|_{L^{2}(\omega)}+\left\|\frac{\partial y_{u^{\star}}}{\partial t}(T)-z_{2}^{d}\right\|_{L^{2}(\omega)}=3.005 \times 10^{-5}$

and cost

$$
\left\|u^{\star}\right\|_{L^{2}(0, T)}=4.84 \times 10^{-2} .
$$




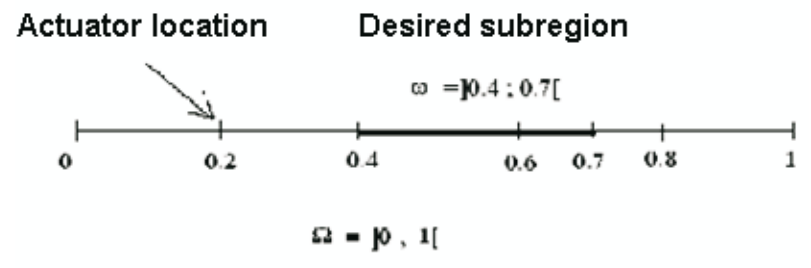

Fig. 5. New actuator location and the new subregion.

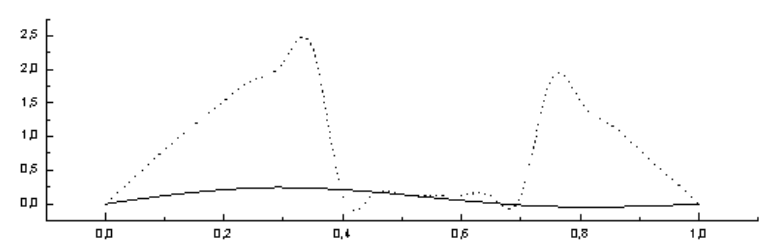

Fig. 6. Desired (dashed line) and final (solid line) position in $\omega$.

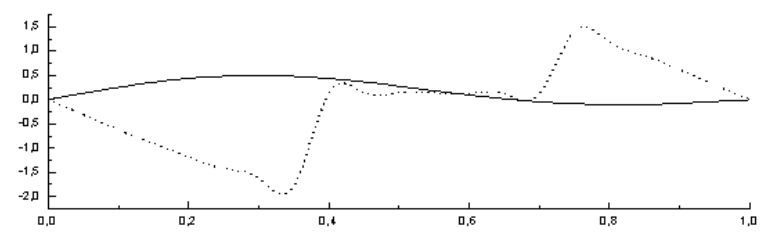

Fig. 7. Desired (dashed line) and final (solid line) speed in $\omega$.

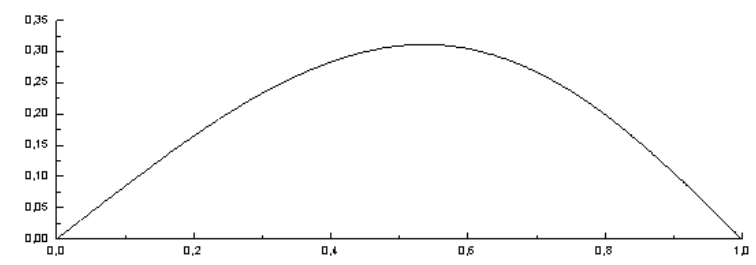

Fig. 8. Evolution of the control function on the time interval $[0, T]$.

\section{Conclusion}

The regional controllability problem for a class of semilinear distributed hyperbolic systems was discussed and solved using linear regional controllability techniques and by applying fixed point theorems. The obtained results lead to an algorithm which was implemented numerically. The method presented in the paper is quite general and covers a wide class of semilinear abstract dynamical systems. Many questions remain open, e.g., the case where the target $\omega$ is a part of the boundary of the system domain. This question is still under consideration and the results will appear in a separate paper.

\section{References}

Brezis H. (1993): Analyse fonctionnelle. Théorie et applications. -Paris: Masson.

Da Prato G. , Pritchard A. and Zabczyk J. (1991): On minimum energy problems. SIAM Journal on Control and Optimization, Vol. 29, pp. 209-221.

De Souza F., J.A.M., and A.J.Pritchard (1985): Control of semilinear distributed parameter systems. Telecommunication and Control, INPE, Sao José dos Campos, Brazil, pp. 160164.

De Souza F. J.A.M (1985): Control of nonlinear ditributed parameter systems. In: Proc. IV Coloquio de Control Automatico (Ibarra-Zannatha, Ed.), Centro de investigation y Estudios Avanzados del instituto Politecnico Nacional de Mexico, Mexico, Vol. 1, pp. 37-43.

El Jai A., Zerrik E., Simon M. C. and Pritchard A. J. (1995): Regional controllability of distributed parameter systems. International Journal of Control, Vol. 62, No. 6, pp. 1351 1365.

Fabre C. , Puel J. P. and Zuazua E. (1995): Approximate controllabiliy of the semilinear heat equation, Proceedings of the Royal Society of Edinburgh, Vol. 125 A, pp. 31-61.

E. (1997): Null controllability of the proximate heat equation, ESAIM: Control Optimization and Calculus of Variations, Vol. 2, pp. 87-103.

Henry D. (1981): Geometric Theory of Semilinear Parabolic Systems.

Kassara K. and El Jaï A. (1983): Algorithme pour la commande d'une classe de systèmes à paramètres répartis non linéaires. Revue marocaine d'automatique, d'informatique et de traitement de signal, Vol. 1, No. 2, pp. 95-117.

Klamka J. (2002): Constrained exact controllability of semilinear systems. Systems and Control Letters, Vol. 47, No. 2, pp. 139-147.

Klamka J. (2001): Constrained controllability of semilinear systems. Nonlinear Analysis, Vol. 47, pp. 2939-2949.

Klamka J. (2000): Schauder's fixed point theorem in nonlinear controllability problem. Control and Cybernetics, Vol. 29, No. 1, pp. 153-165.

Klamka J. (1999): Constrained conllability of dynamical systems. International Journal of Applied Mathematics and Computer Science, Vol. 9, No. 2, pp. 231-244.

Klamka J. (1998): Controllability of second order semilinear infinite-dimensional dynamical systems. Applied Mathematics and Computer Science, Vol. 8, No. 3, pp. 459-470.

Klamka J. (1991): Controllability of Dynamical Systems, Dordrecht: Kluwer Academic Publishers.

Lions J.L. (1988): Contrôlabilité Exacte. Perturbations et Stabilisation des Systèmes Distribués, Tome 1, Contrôlabilité Exacte. -Paris: Masson.

Pazy A. (1983): Semigroups of Linear Operators and Applications to Partial Differential Equations. -Berlin: SpringerVerlag. 
Zeidler E. (1990): Nonlinear Functional Analysis and Its Applications II/A. Linear Applied Functional Analysis, Springer.

Zuazua E. (1990): Exact controllability for the semilinear wave equation. Journal de Mathématiques Pures et Appliquées, 69, pp. 1-31.

Zeidler E. (1999): Applied Functional Analysis. Applications to Mathematical Physics. - Springer.

Zerrik E., Bourray H. and El Jai A. (2004): Regional observability of semilinear distributed parabolic systems. International Journal of Dynamical and Control Systems, Vol. 10, No. 3, pp. 413-430.

Zerrik E. and Larhrissi R. (2002): Regional boundary controllability of hyperbolic systems. Numerical approach. International Journal of Dynamical and Control Systems, Vol. 8, No. 3, pp. 293-311.
Zerrik E. and Larhrissi R. (2001): Regional Target Control of the wave Equation. International Journal of Systems Science, Vol. 32, No. 10, pp. 1233-1242.

Zerrik E., Boutoulout A. and El Jaï A. (2000): Actuators and regional boundary controllability. International Journal of Systems Science, Vol. 31, No. 1, pp. 73-82.

Received: 21 September 2006

Revised: 11 September 2007 